\title{
ZigBee's Received Signal Strength and Latency Evaluation under Varying Environments
}

\author{
H. H. R. Sherazi, ${ }^{1}$ Razi Iqbal, ${ }^{2}$ Sana U1 Hassan, ${ }^{3}$ \\ M. H. Chaudary, ${ }^{4}$ and Syed Asfandyar Gilani ${ }^{5}$ \\ ${ }^{1}$ Department of Electrical \& Information Engineering (DEI), Politecnico di Bari, Via Orabona 4, 70125 Bari, Italy \\ ${ }^{2}$ American University in the Emirates, Dubai Academic City, Dubai 31624, UAE \\ ${ }^{3}$ Lahore Leads University, Kamahan Road, Lahore 54000, Pakistan \\ ${ }^{4}$ Department of Computer Science, COMSATS Institute of Information Technology, Lahore 54000, Pakistan \\ ${ }^{5}$ Department of MIS, City University College, Ajman 18484, UAE
}

Correspondence should be addressed to H. H. R. Sherazi; sherazi@poliba.it

Received 5 April 2016; Accepted 29 May 2016

Academic Editor: Rui Zhang

Copyright (C) 2016 H. H. R. Sherazi et al. This is an open access article distributed under the Creative Commons Attribution License, which permits unrestricted use, distribution, and reproduction in any medium, provided the original work is properly cited.

\begin{abstract}
Being self-configured, self-organized, and self-healing low power technology, ZigBee has obtained significant attention in last few years for achieving ubiquitous communication among various devices within a Personal Area Network (PAN). Even after a decade of its emergence, it has been well serving the communication needs of numerous modern applications belonging to multiple industries and is still a spotlight for the researchers working on certain aspects to enhance productivity along with a major cost reduction. Despite its robust communication nature, it heavily depends upon the context and is prone to the external effects that may cause a serious threat to prospective applications. This paper presents the novel experimental analysis conducted on real test beds to evaluate the impact of continuously changing communication environment on various parameters, for example, RSSI (Received Signal Strength Indicator) and latency in the presence of multiple obstacles that may lead to severe degradation in the overall performance. Eventually, we suggest a suitable frame size for ZigBee based on our results deduced from the experimental study.
\end{abstract}

\section{Introduction}

Modern communication systems have engrossed the troublesome handling and usage of wires in communication networks and promoted the idea of wireless network with time. This emergence gave birth to a number of complexities and entanglements under different circumstances. With the rapid growth of wireless technology, Wireless Sensor Networks (WSNs) were recognized as a convalescent form of wireless mechanism. Therefore, this need led to a technology with peculiarity of low power consumption, high amount of reliability, and efficiency in addition to the cost effectiveness. IEEE 802.15.4 and ZigBee were specially designed for WSN and Wireless Network Monitoring (WNM) [1]. In lieu of high data rate and more functionality, ZigBee is considered popular in all the eyes because of its dramatic low depletion of power that yields low cost and high efficiency [2-6]. ZigBee is the exceptional wireless technology that uses $2.4 \mathrm{GHz}$ and sub-GHX-frequency bands [7]. From factories to the home automation, this technology offers a wide range of applications and the deployment in the various areas like healthcare, agriculture, and education.

This technology has proven to be the new horizon for researchers having curiosity in the field of wireless radio networks. Several applications based on ZigBee have already been introduced by exploiting all the ebb and flow of this technology. ZigBee performance assessment based on different set of grades helps the researchers to get about the suitability of this technology suitable for the distinct zones of applications.

ZigBee has been widely used in recent years but adoption of this technology in home automation is somehow slower [8] as it has its own pros and cons in different areas of specification. For example, it is reliable as far as battery 
life and efficiency are concerned, but it may not be reliable keeping in view the signal strength. It could be used for distance measurements based on its RSSI value. But the value of ZigBee RSSI oscillates with a minor change in environment around it and this fluctuation results in disreputability of this technology.

The major aim of this paper is to study abrupt changes in ZigBee RSSI under varying environment. The average of resulting values could give an approximate and imprecise outcome tolerating different surroundings. Along the RSSI value of ZigBee, measuring the total time taken by certain size of data string can also be served for noticing the latency. Time taken by a specific packet is important for different applications like traffic control system to calculate the distance between two corresponding vehicles.

ZigBee IEEE 802.15.4 is designed for reliable network control and network monitoring as compared to other short range wireless communication technologies like $\mathrm{Wi}-\mathrm{Fi}$, Bluetooth, and ultrawideband [2, 8]. All these technologies utilize lower power but ZigBee devices consume substantially lesser amount of power. Security and integrity of data in ZigBee devices persuade researchers looking at it in a different way. For last few years, many real world applications were designed using this technology and researchers have substantial curiosity in scrutinization, evaluation, and real time deployment of ZigBee protocols [9].

Different indoor experiments have been conducted for varying circumstances and indoor objects have been identified that affect the signal strength of ZigBee: wooden furniture, concrete walls, and people around. By changing the number of these items, we could observe the slight and lofty change in RSSI value. Furthermore, certain sizes of data strings were sent from one ZigBee device to be received on the other end. It is repeated by keeping the distance constant but changing the environment for a precise analysis of latency which resulted in obtaining different values. Then, variable distance between communicating nodes also led to the change in the RSSI value and total latency. Performing the same set of experiments using constant context but extending the distance this time yielded another set of results to be noted. A number of experiments were performed at a sequence of increased distance among the devices.

The remainder of this paper is organized as follows: the next section offers an overview of the current state of the art of ZigBee. Section 3 presents the proposed methodology to be followed for the evaluation of possible scenarios, while the experimental setup describing the various components used for designing real test beds is elaborated in Section 3.1 and results taken from the experiments are analyzed and discussed in Section 3.2. And finally concluding remarks are stated in Section 4.

\section{Literature Review}

ZigBee technology is responsible for the security, reliability, and the long lasting battery life but it carries two types of limitations, that is, bandwidth and range. Also reflection, scattering, and residence of other physical obstacles have adverse impacts on RSSI value of ZigBee. Researchers have conducted several evaluation experiments through ZigBee (IEEE 802.15.4). In [9], the authors deal with the affected Bit Error Rate (BER) and Signal to Noise Ratio (SNR) of ZigBee when there was a change in parameters like Noise Power Value, Data Rate, and Bit Per Symbol.

In [10], Hyncica et al. evaluated the results on the basis of series of experiments and claim that effective range of ZigBee is approximately 12 meters through many dry walls and data rate using SMAC is up to $165 \mathrm{kbps}$ under supreme condition. In our humble opinion and observation, the applications implementing ZigBee technology would have to experience not only the number of walls as described in [11] but also the number of humans and other common obstacles like various types of furniture items in furnished homes and different alike places as it has inauspicious effect on efficiency of ZigBee devices. After performing a sequence of experiments, a careful analysis reflected a vital change in the RSSI and affected range of ZigBee devices in the presence of additionally mentioned items (not covered in [11]) and by the same change, consequently, the latency rate was compromised which will be comprehensively discussed in the following sections.

In [12], they emphasized that ZigBee RSSI is serviceable for location sensing if the compromised value of accuracy is $3-5 \mathrm{~m}$. The RSSI has been tested through varying counts of human presence, glass, and wood. The study was helpful to understand the estimated average change in RSSI value through certain range and distance.

In [13], the Effective Data Rate (EDR) and Packet Delivery Ratio (PDR) in a ZigBee network are presented and claimed that the increase in the number of nodes in network decreases the throughput in a beacon-enabled mode. A graph showed acute deviation in EDR and delivery ratio by escalating nodes because of accumulation in collision probability in CSMACA access medium.

Mobility of ZigBee device observed in [14] and the performance of IEEE 802.15.4 was considered with different mobility cases and indicates that mobility of ZigBee nodes in network raises plentiful inconsistency results as compared to static nodes of network. A lot of packet loss occurs in the case of device mobility and it enlarges as the speed of device movement increases. They observed PDR by applying two types of topologies, mesh routing and tree routing. Packet delivery and efficiency of ZigBee accept various kinds of impact by mobility but in our case we took static nodes in network but especially focused on mobility and change of obstacles and their numbers, and we observed the effect on RSSI, distance, and frame size on the latency rate of data.

RSSI measurement of ZigBee is critical for the localization applications and estimating the position. Ruminating the article [12], we performed series of examinations to evaluate the effect on the latency rate of data sent by ZigBee coordinator by certain size of frame under affected RSSI value. The applications not tolerating the change in latency rate would consider this research to be helpful to actually recognize the aggregate effects on the latency introducing variable data frames sent by a ZigBee coordinator to end nodes and vice versa. As per various studies conducted already, it is a known fact that increase in the distance in communication range 
between the ZigBee modules decreases the signal strength; this study is another effort to further analyze the parameters that actually affect the ZigBee communication in an indoor environment rather than using it outside.

\section{Methodology}

Signal strength of ZigBee radio is greatly affected by the presence of different kind of physical objects. The purpose is to design the scenarios including the obstacles while performing experiments to actually test the performance of ZigBee because it is significant to consider the adverse effects under the presence of these objects while deploying ZigBee technology in home appliances and other indoor applications. All experiments have been conducted in the presence of few possible objects that could attenuate the signal strength caused by scattering and reflection of signals affecting the performance of ZigBee by weakening the RSSI. Major physical objects considered are different numbers of walls, furniture, and humans being that are the most common factors ZigBee experiences while having indoor communication with other nodes.

In the existence of these mentioned objects and varying the count of these obstacles, a series of experiments were performed and, by getting the results, an estimation of affecting proportion in ZigBee RSSI has been made to conclude the best, average, and worst scenarios for ZigBee indoor communication concerning distance and relative change in RSSI and time to receive a certain frame under different obstacles. A varying setup was designed to perform testing experiments, every time with an increase in the distance between two ZigBee nodes while communicating, called case $X$. Five different cases were implemented and each case has further three scenarios. In each scenario, the number of obstacles varies randomly. Taking the overall average of scenarios would be considered appropriate in the indoor performance of ZigBee.

3.1. Experimental Setup. Overall two ZigBee devices were used; both were at API mode while performing experiments to send and receive a set of frames, connected to a computer to monitor the change in RSSI and time difference to find the latency rate. ZigBee network coordinator is a fixed and main device, connected to computer to communicate and receive data from other nodes as shown in Figure 1. End node is the other device connected to a laptop so that it is moveable. Set of frames sent to the coordinator by this node for monitoring at main device is also shown in Figure 1.

In real time indoor scenario, the number of obstacles varies with change in distance as illustrated in Figure 1. The dark highlighted area of 30 feet is effective ZigBee range area where the number of packet losses was minimum. Further 30 - to 45-foot area is weak RSSI area where a significant packet loss was observed. The count of obstacles varied in each scenario under Case 1 using distance of 10 feet, which is illustrated in Table 1.

In Case 1, the distance between two nodes was fixed to 10 feet; the tests of RSSI and time to receive packet were
TABLE 1: Case 1 of experimental setup.

\begin{tabular}{lcccc}
\hline Distance & Scenario & Walls & Furniture & Human \\
\hline \multirow{3}{*}{ 10 feet } & 1 & 4 & 5 & 2 \\
& 2 & 3 & 3 & 2 \\
& 3 & 2 & 2 & 1 \\
\hline
\end{tabular}

repeated in three different scenarios. In first scenario, total number of walls surrounded was 4 , number of furniture chosen was 5, and humans count was 2 in total. A set of results were collected under this scenario. In the next scenario, the number of walls was decreased to 3 by changing the location of nodes and number of furniture pieces were reduced to 3 keeping the number of humans and distance constant, and results were slightly different. Third scenario represents a number of 2 of walls and furniture pieces but humans count was reduced to 1 this time. Under all the three scenarios, data packets bearing variable size were sent to main node from all other nodes and experiment was repeated three times in each scenario to ensure the preciseness. The results taken are shown in Table 2.

In each scenario of Case 1 , the results showed variance in the values taken. The value of RSSI usually increased by decreasing the number of obstacles. It was maximum in scenario 3 and minimum in scenario 1 at 10 -foot distance, but the average RSSI value was $-67 \mathrm{dBm}$. Frames of three different sizes were sent and received at the main node, every time a variation in latency can be noticed in Table 2 where average time taken by a frame of 15 bytes was 0.05 sec. Similarly, a frame of size 50 bytes took slightly less than double the time taken by a frame in scenario 1, being more than three times in size of previous frame. A frame of 100 bytes (double the 50-byte size) took almost $50 \%$ extra time to be received in comparison with 50 -byte frame.

Table 3 illustrates the experiments conducted for Case 2 in which distance was increased to 20 feet with varying number and type of obstacles. As mentioned earlier, obstacles are kept constant to walls, furniture, and humans. No new obstacles were introduced to avoid confusion between effects of obstacles on ZigBee communication. Table 3 illustrates the experimental setup for Case 2 in an indoor environment.

It shows that there were the same number of walls and furniture pieces but numbers of humans were 3 and distance doubled in first scenario of Case 2. Count of all types of obstacles decreased except for furniture in the second scenario. Third scenario consists of the least number of walls and human's presence. Under these conditions, the results were obtained and are illustrated in Table 4.

The average RSSI in Case 2 was $-77 \mathrm{dBm}$ but the maximum RSSI gained at distance 20 feet was in scenario 3 of this case, where number of walls and humans was the least. Packet loss was not significant and average time taken by frames of 15 bytes was approximately the same as in Case 1. Similarly, there were no variations noticed in frame of sizes 50 bytes and 100 bytes. The 50 -byte frame time was even better than that of Case 1.

Table 6 illustrates the experiments conducted for Case 3 in which distance was increased to 30 feet with varying 


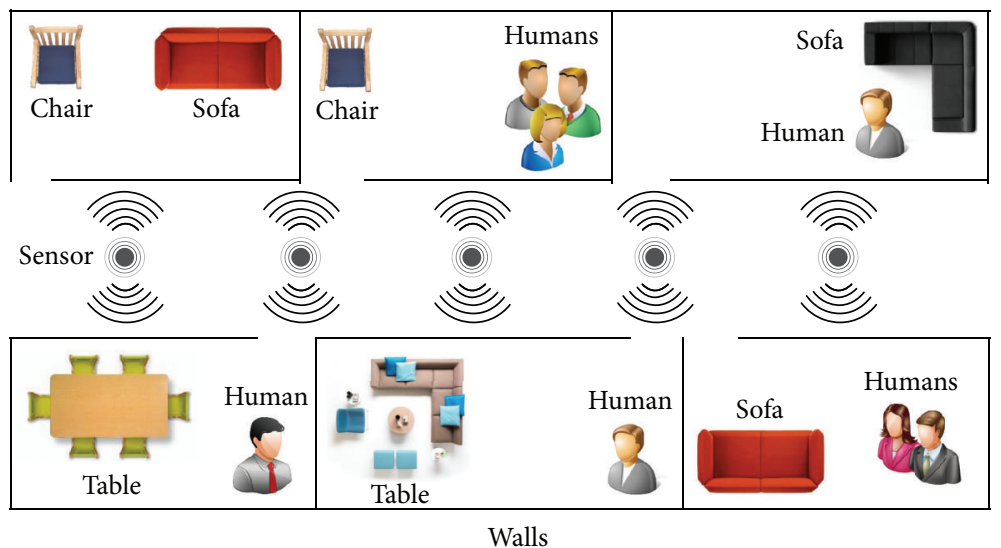

FIGURE 1: System model to represent indoor location and devices placement.

TABLE 2: Experimental results of Case 1.

\begin{tabular}{|c|c|c|c|c|c|c|c|c|}
\hline \multirow{3}{*}{ Scenario } & \multicolumn{8}{|c|}{ Frame received time (s) } \\
\hline & \multicolumn{2}{|c|}{ RSSI (dBm) } & \multicolumn{2}{|c|}{15 bytes } & \multicolumn{2}{|c|}{50 bytes } & \multicolumn{2}{|c|}{100 bytes } \\
\hline & RSSI & Average & Time & Average & Time & Average & Time & Average \\
\hline \multirow{4}{*}{1} & \multirow{3}{*}{-71} & \multirow{9}{*}{-67} & 0.038 & \multirow{9}{*}{0.05} & 0.101 & \multirow{9}{*}{0.093} & 0.160 & \multirow{9}{*}{0.142} \\
\hline & & & 0.033 & & 0.075 & & 0.115 & \\
\hline & & & 0.053 & & 0.100 & & 0.130 & \\
\hline & \multirow{3}{*}{-68} & & 0.067 & & 0.107 & & 0.183 & \\
\hline \multirow[t]{3}{*}{2} & & & 0.055 & & 0.080 & & 0.160 & \\
\hline & & & 0.046 & & 0.092 & & 0.101 & \\
\hline & & & 0.066 & & 0.094 & & 0.165 & \\
\hline \multirow[t]{2}{*}{3} & -64 & & 0.056 & & 0.096 & & 0.136 & \\
\hline & & & 0.040 & & 0.097 & & 0.132 & \\
\hline
\end{tabular}

TABLE 3: Case 2 of experimental setup.

\begin{tabular}{lcccc}
\hline Distance & Scenario & Walls & Furniture & Human \\
\hline \multirow{3}{*}{20 feet } & 1 & 4 & 5 & 3 \\
& 2 & 3 & 6 & 2 \\
& 3 & 2 & 5 & 1 \\
\hline
\end{tabular}

TABLE 4: Experimental results of Case 2.

\begin{tabular}{|c|c|c|c|c|c|c|c|c|}
\hline \multirow{3}{*}{ Scenario } & \multicolumn{8}{|c|}{ Frame received time (s) } \\
\hline & \multicolumn{2}{|c|}{ RSSI (dBm) } & \multicolumn{2}{|c|}{15 bytes } & \multicolumn{2}{|c|}{50 bytes } & \multicolumn{2}{|c|}{100 bytes } \\
\hline & RSSI & Average & Time & Average & Time & Average & Time & Average \\
\hline \multirow{3}{*}{1} & \multirow{3}{*}{-80} & \multirow{6}{*}{-77} & 0.045 & \multirow{9}{*}{0.052} & 0.094 & \multirow{9}{*}{0.092} & 0.129 & \multirow{9}{*}{0.144} \\
\hline & & & 0.052 & & 0.109 & & 0.131 & \\
\hline & & & 0.075 & & 0.087 & & 0.156 & \\
\hline \multirow{3}{*}{2} & \multirow{3}{*}{-78} & & 0.050 & & 0.100 & & 0.146 & \\
\hline & & & 0.062 & & 0.091 & & 0.142 & \\
\hline & & & 0.063 & & 0.078 & & 0.167 & \\
\hline \multirow{3}{*}{3} & \multirow{3}{*}{-74} & & 0.040 & & 0.091 & & 0.131 & \\
\hline & & & 0.038 & & 0.077 & & 0.152 & \\
\hline & & & 0.046 & & 0.110 & & 0.150 & \\
\hline
\end{tabular}


TABLE 5: Case 3 of experimental setup.

\begin{tabular}{lcccc}
\hline Distance & Scenario & Walls & Furniture & Human \\
\hline \multirow{3}{*}{30 feet } & 1 & 5 & 7 & 3 \\
& 2 & 3 & 5 & 2 \\
& 3 & 3 & 3 & 1 \\
\hline
\end{tabular}

TABLE 6: Experimental results of Case 3.

\begin{tabular}{|c|c|c|c|c|c|c|c|c|}
\hline \multirow{3}{*}{ Scenario } & \multicolumn{8}{|c|}{ Frame received time (s) } \\
\hline & \multicolumn{2}{|c|}{ RSSI (dBm) } & \multicolumn{2}{|c|}{15 bytes } & \multicolumn{2}{|c|}{50 bytes } & \multicolumn{2}{|c|}{100 bytes } \\
\hline & RSSI & Average & Time & Average & Time & Average & Time & Average \\
\hline \multirow{4}{*}{1} & \multirow{3}{*}{-86} & \multirow{9}{*}{-82} & 0.037 & \multirow{9}{*}{0.052} & 0.072 & \multirow{9}{*}{0.081} & 0.139 & \multirow{9}{*}{0.153} \\
\hline & & & 0.050 & & 0.087 & & 0.149 & \\
\hline & & & 0.041 & & 0.092 & & 0.150 & \\
\hline & \multirow{3}{*}{-84} & & 0.046 & & 0.088 & & 0.160 & \\
\hline \multirow[t]{3}{*}{2} & & & 0.061 & & 0.077 & & 0.156 & \\
\hline & & & 0.060 & & 0.087 & & 0.160 & \\
\hline & \multirow{3}{*}{-78} & & 0.052 & & 0.074 & & 0.141 & \\
\hline \multirow[t]{2}{*}{3} & & & 0.065 & & 0.078 & & 0.160 & \\
\hline & & & 0.056 & & 0.080 & & 0.165 & \\
\hline
\end{tabular}

TABLE 7: Case 4 of experimental setup.

\begin{tabular}{lcccc}
\hline Distance & Scenario & Walls & Furniture & Human \\
\hline \multirow{3}{*}{40 feet } & 1 & 5 & 7 & 3 \\
& 2 & 3 & 5 & 2 \\
& 3 & 3 & 3 & 1 \\
\hline
\end{tabular}

number and type of obstacles. Like Case 2, type of obstacles is kept the same to avoid confusion.

Table 5 illustrates the experimental setup for Case 3 in an indoor environment. In the first scenario, numbers of walls, furniture pieces, and humans are increased. In the second scenario, the location was shifted to decrease the number of walls but distance kept the same and the number of furniture pieces and humans reduced. Third scenario consists of decrease in count of furniture; humans and walls were the same and the results obtained in this case are described in Table 6.

The maximum RSSI values in Case 3 were in scenario 3 where there was minimum number of walls and furniture but average RSSI obtained was $-82 \mathrm{dBm}$. Packets loss in this case was slightly greater then Cases 1 and 2 . Frame of 15 bytes took the same average time to receive but 50-byte frame showed a dramatic increase of time; also 100-byte frame took a little bit more time in this case.

Beyond 30 feet the RSSI value of ZigBee got weakened. Table 8 illustrates the experiments conducted for Case 4 in which distance was increased to 40 feet with varying number and types of obstacles. Like Case 3 types of obstacles are kept the same to avoid confusion.

Table 7 illustrates the experimental setup for Case 4 in an indoor environment. In this case with distance of 40 feet, count of obstacles was the same as in previous case in all three scenarios and the results obtained are shown in Table 8.
The maximum value of RSSI was observed in third scenario in this case, which was around $-83 \mathrm{dBm}$ but the average RSSI was $-86 \mathrm{dBm}$ under all scenarios. The amount of packet loss was greater in this case as compared to previous cases but time taken by a frame of 15 bytes was the same again. A dramatic increase of latency rate was observed in frame of size 50 bytes and it took average $0.1 \mathrm{sec}$ to be received. Also 100 -byte frame took $0.15 \mathrm{sec}$ for the reception.

Case 5 consists of maximum range distance, which is 45 feet, and details regarding number of obstacles are shown in Table 9. In this case, the number of walls was 6 with the number of furniture pieces equal to 7 and humans count being 3 under scenario 1 . Count of obstacles decreased by each scenario. The results obtained this time are described in Table 10.

$60 \%$ of packets were lost in this case because of the placement of end node at extreme distance and average RSSI values obtained were as weak as $-89 \mathrm{dBm}$. The 15 -byte frame took the least time as compared to all other cases. Average time was $0.067 \mathrm{sec}$ but 50 -byte frame was as usual unpredictable and it took reasonably average time than other cases and was received in $0.093 \mathrm{sec}$. 100-byte frame took a lot more time that was on average $0.182 \mathrm{sec}$.

3.2. Experimental Result Analysis. Scattering and disturbance of signal put relative impact on ZigBee performance. Each time variation in RSSI with little change in indoor environment and their corresponding time deviation reveals a strong instability of ZigBee signals and latency rate. Results show that a single frame of different size took noticeably different time. Doubling the size of frame does not affect more the latency rate if the distance is the same and whatever the number of obstacles is. But increase in distance actually increases the relative time of two different sizes of frames. A dramatic increase of time occurred when end node was at 
TABLE 8: Experimental results of Case 4.

\begin{tabular}{|c|c|c|c|c|c|c|c|c|}
\hline \multirow{3}{*}{ Scenario } & \multicolumn{8}{|c|}{ Frame received time $(\mathrm{s})$} \\
\hline & \multicolumn{2}{|c|}{ RSSI (dBm) } & \multicolumn{2}{|c|}{15 bytes } & \multicolumn{2}{|c|}{50 bytes } & \multicolumn{2}{|c|}{100 bytes } \\
\hline & RSSI & Average & Time & Average & Time & Average & Time & Average \\
\hline \multirow{3}{*}{1} & \multirow{3}{*}{-88} & \multirow{6}{*}{-86} & 0.031 & \multirow{9}{*}{0.05} & 0.041 & \multirow{9}{*}{0.081} & 0.139 & \multirow{9}{*}{0.154} \\
\hline & & & 0.046 & & 0.089 & & 0.149 & \\
\hline & & & 0.046 & & 0.109 & & 0.150 & \\
\hline \multirow{3}{*}{2} & \multirow{3}{*}{-85} & & 0.042 & & 0.092 & & 0.160 & \\
\hline & & & 0.051 & & 0.091 & & 0.156 & \\
\hline & & & 0.061 & & 0.121 & & 0.160 & \\
\hline \multirow{3}{*}{3} & \multirow{3}{*}{\multicolumn{2}{|c|}{-83}} & 0.056 & & 0.074 & & 0.160 & \\
\hline & & & 0.056 & & 0.078 & & 0.175 & \\
\hline & & & 0.067 & & 0.080 & & 0.165 & \\
\hline
\end{tabular}

TABLE 9: Case 5 of experimental setup.

\begin{tabular}{lcccc}
\hline Distance & Scenario & Walls & Furniture & Human \\
\hline \multirow{3}{*}{40 feet } & 1 & 5 & 7 & 3 \\
& 2 & 3 & 5 & 2 \\
& 3 & 3 & 3 & 1 \\
\hline
\end{tabular}

extreme distance position. The value of RSSI was affected in almost the same amount at each distance. Table 11 represents the overall RSSI values and time latency at all observed distances.

In Table 11, it can be seen that the time of smaller frame of 15 bytes is almost the same at all distances except for the slight difference at extreme distance of 45 feet. The behavior of 50-byte frame size is relatively unpredictable throughout the cases and the shortest time taken by this size of frame is in case of distance of 30 feet as compared to other distances and RSSI value is $-89 \mathrm{dBm}$ even for the extreme distance as shown in Table 11. 100-byte frame size shows consistent increase in time with increase in distance and it is almost $1 / 5$ th of a sec even in extreme case.

A relationship graph between number of obstacles and their corresponding values of RSSI is shown in Figure 2. The maximum value of RSSI observed is around $-64 \mathrm{dBm}$ at distance of 10 feet. Then it is gradually decreasing as the number of obstacles keeps on increasing. Similar effect can be seen in each case. The distance of 10 and 40 feet gives a slight but constant decrease in RSSI value relative to the number of obstacles. It is showing a deviation and making a curve in other cases, which shows irregular change in RSSI value.

A graph of distance versus time taken by a small, medium, and large sized frame is shown in Figure 3.

Time for 15-byte frame remains constant till distance of 40 feet but it actually increases at distance of 45 feet, which shows that, for using very small data or small decision-making bits, the distance up to 40 feet is reasonably good for any sized frame under any number of obstacles from zero to 16. Sending a medium size frame of 50 bytes took about the same time at the distance of 10 and 20 feet and tends to decrease afterwards till it reaches 30 feet because of the number of obstacles. It again shows an elevation behavior between 30

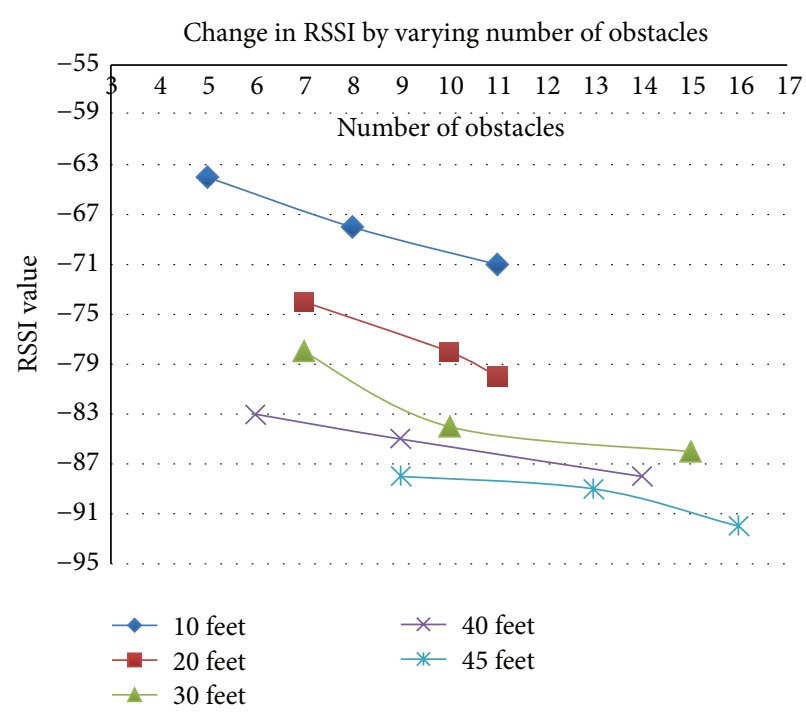

FIGURE 2: Effect on RSSI value by increasing number of obstacles.

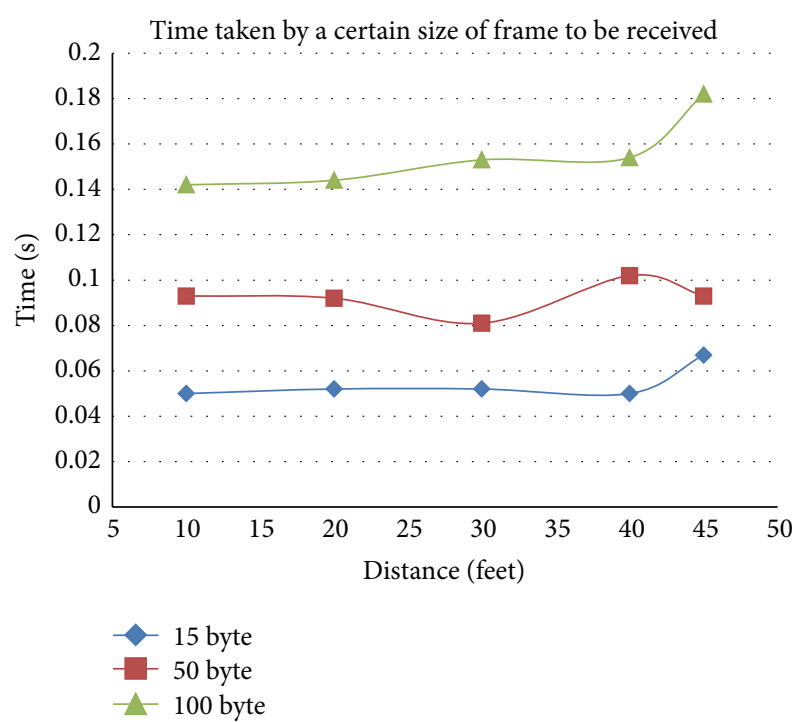

FIGURE 3: Frame receiving time by increasing distance for different sized frames. 
TABLE 10: Experimental results of Case 5.

\begin{tabular}{|c|c|c|c|c|c|c|c|c|}
\hline \multirow{3}{*}{ Scenario } & \multicolumn{8}{|c|}{ Frame received time (s) } \\
\hline & \multicolumn{2}{|c|}{ RSSI (dBm) } & \multicolumn{2}{|c|}{15 bytes } & \multicolumn{2}{|c|}{50 bytes } & \multicolumn{2}{|c|}{100 bytes } \\
\hline & RSSI & Average & Time & Average & Time & Average & Time & Average \\
\hline \multirow{3}{*}{1} & \multirow{3}{*}{-92} & \multirow{6}{*}{-89} & 0.073 & \multirow{9}{*}{0.067} & 0.087 & \multirow{9}{*}{0.093} & 0.171 & \multirow{9}{*}{0.182} \\
\hline & & & 0.065 & & 0.079 & & 0.180 & \\
\hline & & & 0.061 & & 0.101 & & 0.186 & \\
\hline \multirow{3}{*}{2} & \multirow{3}{*}{-89} & & 0.067 & & 0.103 & & 0.180 & \\
\hline & & & 0.068 & & 0.090 & & 0.181 & \\
\hline & & & 0.071 & & 0.100 & & 0.190 & \\
\hline \multirow{3}{*}{3} & \multirow{3}{*}{-88} & & 0.057 & & 0.094 & & 0.191 & \\
\hline & & & 0.069 & & 0.098 & & 0.169 & \\
\hline & & & 0.075 & & 0.091 & & 0.192 & \\
\hline
\end{tabular}

TABLE 11: Overall performance evaluation at various distances.

\begin{tabular}{lccccc}
\hline Scenario & Distance (feet) & $\begin{array}{c}\text { Average number of } \\
\text { obstacles }\end{array}$ & Average RSSI & \multicolumn{2}{c}{ Frame received time (s) } \\
\hline 1 & 10 & 8 & -67 & 0.05 & 0.09 \\
2 & 20 & 9 & -77 & 0.05 & 0.09 \\
3 & 30 & 11 & -82 & 0.05 & 0.08 \\
4 & 40 & 10 & -86 & 0.05 & 0.14 \\
5 & 45 & 13 & -89 & 0.06 & 0.15 \\
\hline
\end{tabular}

and 40 feet. A 100-byte frame took almost double the time of 50 bytes but behavior shows that latency increases linearly while increasing the distance showing a constant behavior that prevails till 40 feet. Then, it bears a sudden increase at an extreme distance of 45 feet, so was the case of 15-byte frame.

The results conclude that small sized and full sized frames of ZigBee take relatively more time at extreme distances and remain almost constant at feasible distances. The behavior of medium sized frame is noticeable, and it remains in a range of $0.08 \mathrm{sec}$ (i.e., lowest time) and $0.10 \mathrm{sec}$ (i.e., highest time) that is at the distance of 30 feet and 40 feet, respectively. Thus a medium sized frame is most reliable as long as the performance is concerned under any distance and number of obstacles.

\section{Conclusion}

ZigBee is very useful technology for Wireless Sensor Networks (WSN) because of its features of reliability and efficiency in performance, yet the limitations stand with this technology. The signal strength of ZigBee is highly prone to external effects of the context in which the communication is taking place. It, somehow, affects the latency of data packet and performance in different ways. It could be implemented in different applications that are solely based on its RSSI. To analyze this critical signal strength with varying indoor environments and their relative effects on the performance and latency, a series of test experiments have been conducted. The results showed that the effect of obstacles on ZigBee's signal strength is significant in some situations. A small data frame of 15 bytes took on average $0.05 \mathrm{sec}$ while 100 -byte frame took on average $0.15 \mathrm{sec}$ to be successfully received but time dramatically increases at extreme distance. A medium sized frame of 50 bytes showed a random variation in latency in almost all the cases. The time appears to behave normally even at extreme distance for this size, which shows the reliability of medium sized frame of ZigBee under any distance within range and various number of obstacles. This evaluation study aims at providing a comprehensive guideline to the possible future research towards wide range of applications sensitive to signal strength and latency.

\section{Competing Interests}

The authors declare that there are no competing interests regarding the publication of this paper.

\section{References}

[1] J. T. Adams, "An introduction to IEEE STD 802.15.4," in Proceedings of the IEEE Aerospace Conference, 8 pages, Big Sky, Mont, USA, March 2006.

[2] J.-S. Lee, Y.-W. Su, and C.-C. Shen, "A comparative study of wireless protocols: bluetooth, UWB, ZigBee, and Wi-Fi," in Proceedings of the 33rd Annual Conference of the IEEE Industrial Electronics Society (IECON '07), pp. 46-51, IEEE, Taipei, Taiwan, November 2007.

[3] J. Baviskar, A. Mulla, M. Upadhye, J. Desai, and A. Bhovad, "Performance analysis of ZigBee based real time Home Automation system," in Proceedings of the International Conference on Communication, Information \& Computing Technology (ICCICT '15), pp. 1-6, Mumbai, India, January 2015. 
[4] M. S. Habtoor and H. Rowaihy, "Performance evaluation of IRIS and LOTUS motes when communicating in real environments," International Journal of Distributed Sensor Networks, vol. 2016, Article ID 7347158, 10 pages, 2016.

[5] W. Du, D. Navarro, and F. Mieyeville, "Performance evaluation of IEEE 802.15.4 sensor networks in industrial applications," International Journal of Communication Systems, vol. 28, no. 10, pp. 1657-1674, 2015.

[6] K.-H. Phung, B. Lemmens, M. Goossens, A. Nowe, L. Tran, and K. Steenhaut, "Schedule-based multi-channel communication in wireless sensor networks: a complete design and performance evaluation," Ad Hoc Networks, vol. 26, pp. 88-102, 2015.

[7] M. Tabassum and K. Zen, "Performance evaluation of ZigBee in indoor and outdoor environment," in Proceedings of the 9th International Conference on IT in Asia (CITA' 15), pp. 1-7, Kota Samarahan, Malaysia, August 2015.

[8] K. Gill, S.-H. Yang, F. Yao, and X. Lu, "A ZigBee-based home automation system," IEEE Transactions on Consumer Electronics, vol. 55, no. 2, pp. 422-430, 2009.

[9] K. Shuaib, M. Alnuaimi, M. Boulmalf, I. Jawhar, F. Sallabi, and A. Lakas, "Performance evaluation of IEEE 802.15.4: experimental and simulation results," Journal of Communications, vol. 2, no. 4, pp. 29-37, 2007.

[10] O. Hyncica, P. Kacz, P. Fiedler, Z. Bradac, P. Kucera, and R. Vrba, "The zigbeeexperience," in Proceedings of the 2nd International Symposium on Communications, Control, and Signal Processing, March 2006.

[11] K. Benkič, M. Malajner, P. Planinšič, and Ž. Čučej, "Using RSSI value for distance estimation in wireless sensor networks based on ZigBee," in Proceedings of the 15th International Conference on Systems, Signals and Image Processing (IWSSIP '08), pp. 303306, Bratislava, Slovakia, June 2008.

[12] K. Subaashini, G. Dhivya, and R. Pitchiah, "ZigBee RF signal strength for indoor location sensing-experiments and results," in Proceedings of the 15th International Conference on Advanced Communication Technology: Smart Services with Internet of Things! (ICACT '13), pp. 50-57, IEEE, PyeongChang, Republic of Korea, January 2013.

[13] J.-S. Lee, "Performance evaluation of IEEE 802.15.4 for lowrate wireless personal area networks," IEEE Transactions on Consumer Electronics, vol. 52, no. 3, pp. 742-749, 2006.

[14] L.-J. Chen, T. Sun, and N.-C. Liang, "An evaluation study of mobility support in ZigBee networks," Journal of Signal Processing Systems, vol. 59, no. 1, pp. 111-122, 2010. 


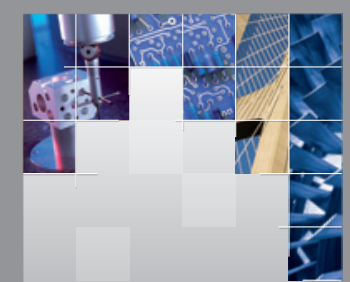

\section{Enfincering}
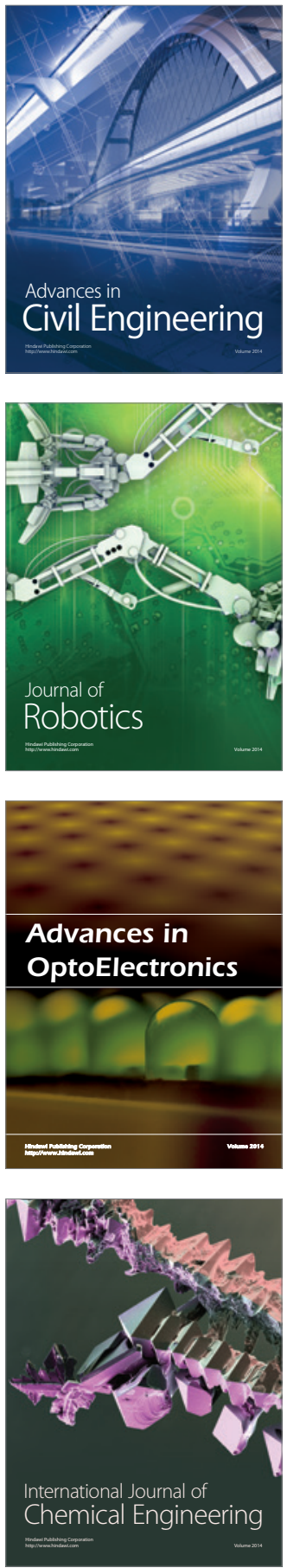

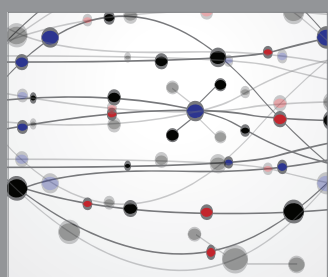

The Scientific World Journal

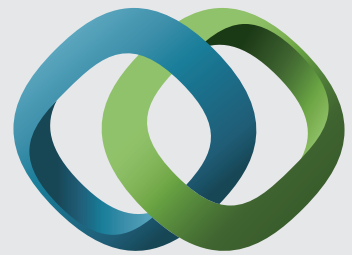

\section{Hindawi}

Submit your manuscripts at

http://www.hindawi.com
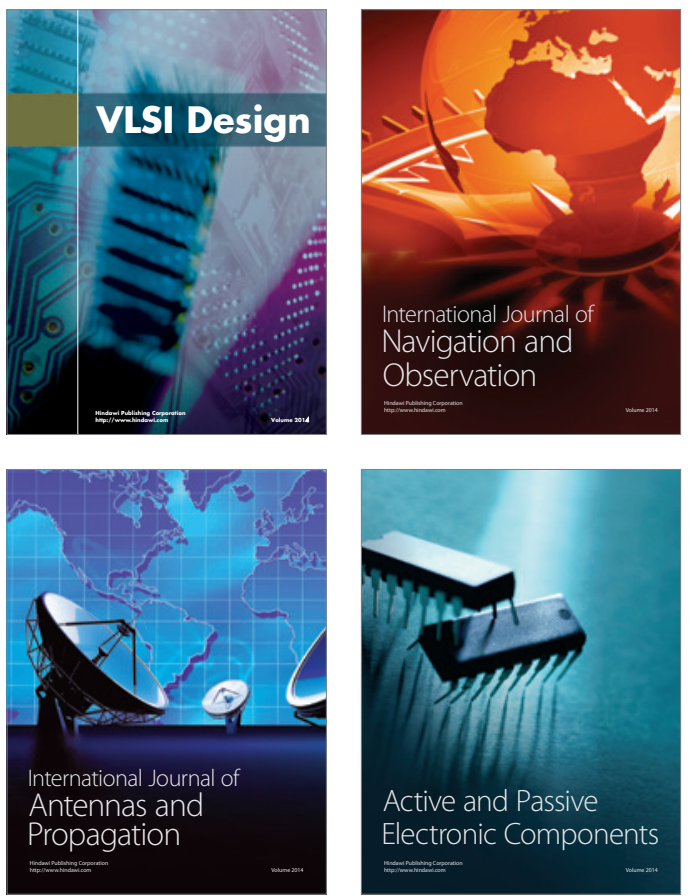
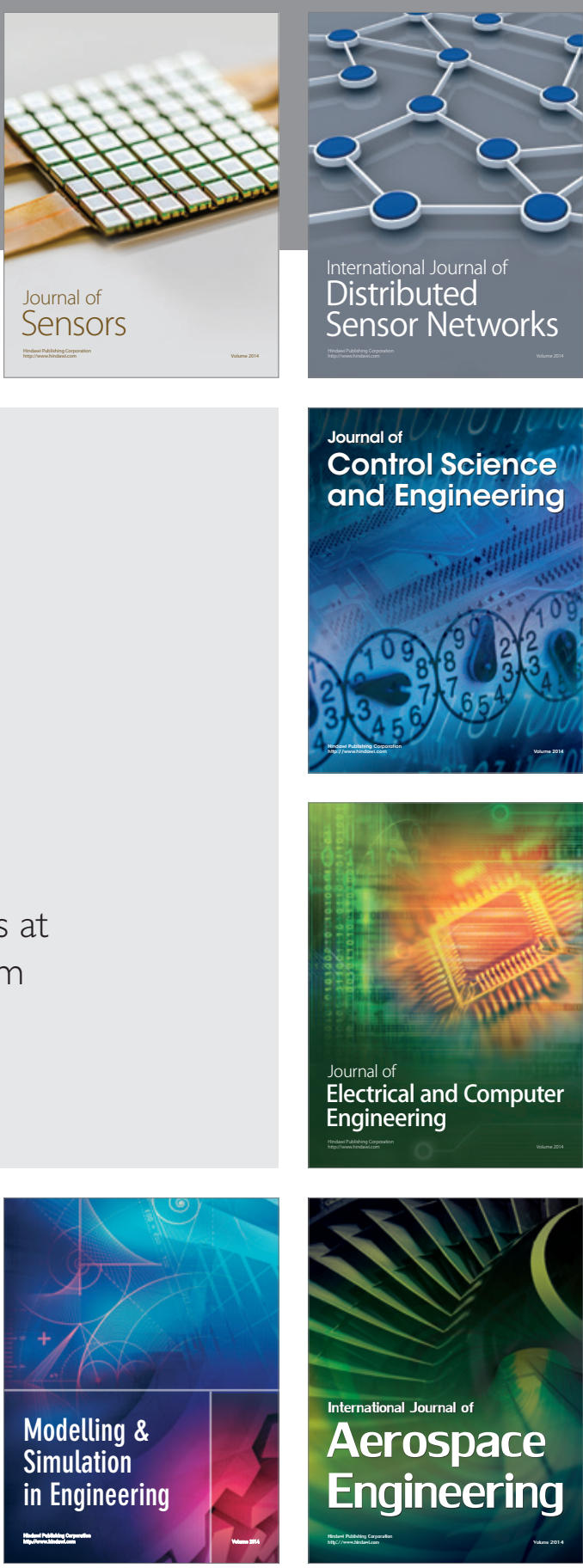

International Journal of

Distributed

Sensor Networks

Journal of

Control Science

and Engineering
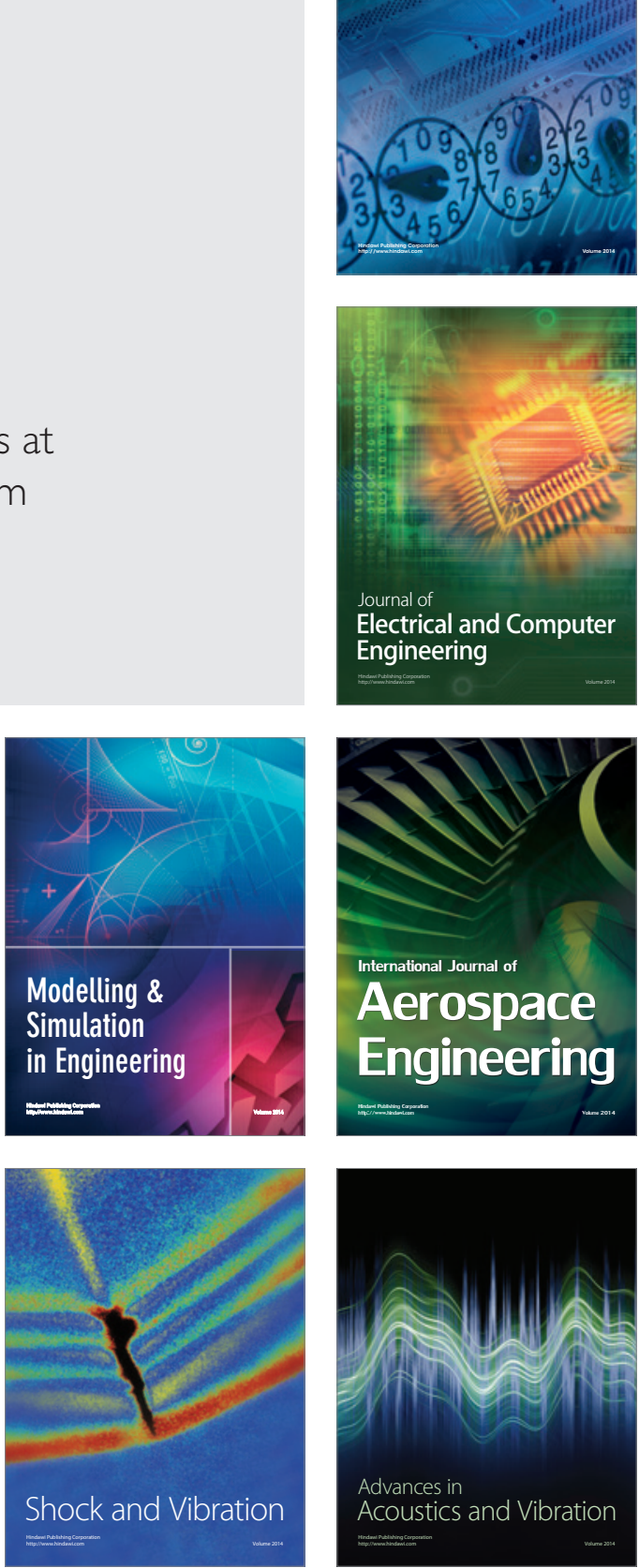